\title{
Molecular-genetic imaging: current and future perspectives
}

\author{
Ronald G. Blasberg and Juri Gelovani Tjuvajev \\ Departments of Neurology and Radiology, Memorial Sloan-Kettering Cancer Center, New York, New York, USA \\ J. Clin. Invest. 111:1620-1629 (2003). doi:10.1172/JCI200318855.
}

Medical imaging has undergone a revolution in the past decade. This is largely due to improved technology involving all the major imaging modalities: MRI, computed tomography (CT), positron emission tomography (PET), ultrasound, and optical imaging. These advances and improvements in technology are being rapidly translated into the clinic and have established new standards of medical practice. Cancer imaging was identified as one of six "extraordinary scientific opportunities" by the National Cancer Institute in 1997-1998, and the institute's subsequent funding initiatives have provided a major stimulus for further developments. A major target of these initiatives has been development of and support for molecular imaging.

Molecular-genetic imaging provides visualization in space and time of normal as well as abnormal cellular processes at a molecular or genetic level. Needless to say, current gamma camera, PET, MRI, and optical technologies do not visualize individual cells, much less molecules. Perhaps the most exciting aspect of this emerging new field are the novel imaging paradigms being developed - paradigms that image molecular-genetic processes rather than anatomy. These paradigms can be successful within the inherent spatial-resolution limits of existing imaging systems, provided that the volume element, or voxel, of the tissue (cells) is relatively homogenous. This review will focus primarily on radionuclide imaging,

Address correspondence to: Ronald G. Blasberg, Department of Neurology and Radiology, Memorial Sloan-Kettering Cancer Center, Box 52, 1275 York Avenue, New York, New York 10021, USA. Phone: (212) 639-7337; Fax: (212) 717-3063;

E-mail: blasberg@neuro1.mskcc.org.

Conflict of interest: The authors have declared that no conflict of interest exists.

Nonstandard abbreviations used: positron emission tomography (PET); fluorodeoxyglucose (FDG); radiolabeled antisense oligonucleotide (RASON); human dopamine 2 receptor $(\mathrm{h} D 2 R)$; human somatostatin receptor subtype 2 (hSSTR2); human sodium iodide symporter (hNIS); 9-(4-fluoro-3hydroxymethylbutyl)guanine (FHBG); 2'-fluoro-2'-deoxy-1- $\beta$-Darabinofuranosyl-5-iodouracil (FIAU); gastrointestinal stromal tumor (GIST); carcinoembryonic antigen (CEA); HSV1-tk and enhanced GFP fusion gene (TKeGFP); N, $\mathrm{N}^{\prime}$-bis(2-chloroethyl)-Nnitrosourea (BCNU); prostrate-specific antigen enhancer (PSE); two-step transcriptional amplification (TSTA); dihydrofolate reductase (DHFR); nuclear factor of activated T cells (NFAT). although many of the principles described are directly applicable to optical and MRI technology as well. A more extensive discussion of these issues was recently published (1).

Molecular imaging has its roots in molecular biology and cell biology as well as in imaging technology. Three different noninvasive, in vivo imaging technologies have developed more or less in parallel: (a) MRI (2-6); (b) nuclear imaging (quantitative autoradiography, gamma camera, and PET) (7-11); and (c) optical imaging of small animals (12-14). The convergence of these disciplines is at the heart of the molecular imaging success story and constitutes the wellspring for further advances in the field. The development of versatile and sensitive assays that do not require tissue samples will be of considerable value for monitoring of molecular-genetic and cellular processes in animal models of human disease, as well as for studies in human subjects. Noninvasive imaging of molecular-genetic and cellular processes will complement established ex vivo molecular-biological assays that require tissue sampling; it also provides a spatial as well as a temporal dimension to our understanding of various diseases.

\section{Imaging strategies}

The three most widely used imaging strategies are direct, indirect, and surrogate. Direct molecular imaging generally involves direct probe-target interaction, whereby the resultant image of probe localization and image intensity is directly related to its interaction with the target epitope or enzyme. This strategy is based on imaging the target directly, usually with a target-specific probe. Indirect molecular imaging is a bit more complex in that it may involve multiple components. One type of indirect imaging that is now being widely used is reporter imaging, which involves a reporter gene and a reporter probe. These components must be complementary; the reporter gene product is frequently an enzyme that converts a reporter probe to a metabolite that is selectively trapped within transduced cells. Alternatively, the reporter gene product can be a receptor or transporter that irreversibly traps the probe in transduced cells during the period of image acquisition. Indirect reporter imaging paradigms are currently 
more widely used than direct molecular imaging studies, particularly in preclinical animal studies, and will be discussed below in greater detail. Surrogateimaging strategies reflect downstream effects of one or more endogenous molecular-genetic processes. This latter approach is particularly attractive for potential translation into clinical studies in the near term, because it uses established radiopharmaceuticals and clinical imaging protocols already in use in the clinic (or soon to be implemented). Examples of direct, indirect, and surrogate imaging using radiolabeled probes and PET to visualize endogenous molecular processes, such as the regulation of endogenous gene expression, will be discussed below.

\section{Direct-imaging strategies}

Direct strategies are used by all three (nuclear, MR, and optical) imaging motifs. For example, mAb- or peptide-specific targeting of a particular cell membrane epitope can be imaged with a paramagnetic, fluorescent, or radionuclide-labeled probe. Imaging of cell surface-specific antigens or epitopes with radiolabeled antibodies is an example of direct molecular imaging that has developed over the past 30 years. Similarly, PET imaging of receptor density or occupancy using small radiolabeled molecular probes has also been widely used, particularly in neuroscience research, over the past two decades. These examples represent some of the first molecular imaging applications used in clinical nuclear medicine research. Other examples of direct-imaging paradigms that use radiolabeled analogues of naturally occurring compounds, such as ${ }^{18} \mathrm{~F}$-fluorodeoxyglucose (FDG) to image glucose utilization in the brain, have been used extensively in the past. FDG PET imaging of glucose utilization is based largely on the activity of the enzyme hexokinase and was described more than two decades ago $(15,16)$. Similarly, imaging of the activity of a particular transporter with a transport-specific probe has been described previously and has been used to image tumors $(17,18)$.

A more recent direct-imaging strategy involves the development of antisense and aptamer oligonucleotide probes that specifically hybridize to target mRNA or proteins in vivo. Radiolabeled antisense oligonucleotides (RASONs) have been developed to directly image endogenous gene expression at the transcriptional level. RASONs are small oligonucleotide sequences that are complementary to a small segment of target mRNA or DNA and that could potentially target any specific mRNA or DNA sequence.In this context, imaging of specific mRNAs with RASONs produces direct images of specific molecular-genetic events. Some efficacy for gamma camera and PET imaging of endogenous gene expression using RASONs has been reported $(19,20)$. Nevertheless, RASON imaging has several serious limitations, including (a) a low number of target mRNA and DNA molecules per cell; (b) limited tracer delivery (poor cell membrane and vascular permeability prevents the tracer from penetrating the blood-brain barrier); (c) poor stability (degradation by H-RNase); (d) slow clearance (slow washout of nonbound oligonucleotides); and (e) comparatively high background activity and low specificity of localization (low target/background ratios). The imaging of specific RASON targets in the body is complicated, and interpretation of the images must be approached with caution.

Direct-imaging strategies are constrained by the necessity to develop a specific probe for each molecular target, and then to validate both sensitivity and specificity in the application of each newly developed probetarget imaging paradigm. This can be very time consuming and costly (e.g., the development, validation, and regulatory approval of ${ }^{18} \mathrm{~F}-\mathrm{FDG}$ PET imaging of glucose utilization in tumors have taken over 20 years). Nevertheless, this traditional approach remains central and is widely used to develop new probes for imaging specific molecular-genetic targets using radiolabeled, paramagnetic, or fluorescent small molecular probes.

\section{Indirect-imaging strategies}

Indirect strategies are used widely for radionuclidebased molecular imaging $(21,22)$ and for optical imaging $(14,23,24)$, and to a lesser degree for $\operatorname{MRI}(5,6)$. Most indirect molecular imaging paradigms involve the use of reporter-transgene technology, which couples a reporter gene with a complementary reporter probe (Figure 1). Imaging the level of reporter gene product activity through probe accumulation provides indirect information that reflects the level of reporter gene expression, and the level of endogenous signaling/transcription factors that drive reporter gene expression. Reporter gene imaging was initially developed and used with in situ optical technology that usually required postmortem tissue sampling and processing (e.g., $\beta$-gal assay). More recent studies have emphasized noninvasive-imaging techniques. Noninvasive reporter imaging is now widely applied in experimental living animals and will soon be applied in human studies. Noninvasive reporter gene imaging involves a reporter transgene (e.g., the herpes simplex virus type 1 thymidine kinase gene HSV1-tk) placed under the control of upstream promoter/enhancer elements. These promoter/enhancer elements can always be "turned on" with constitutive promoters (e.g., long terminal repeat, Rous sarcoma virus, and CMV), or they can be sensitive to activation by specific endogenous transcription factors (factors that bind to and activate specific promoter/enhancer elements). Several noninvasive-imaging paradigms have been described, and it has recently been shown that transcriptional regulation of endogenous gene expression can be imaged using both nuclear (PET) and optical (fluorescence) imaging $(25,26)$. Combined PET and bioluminescence imaging reporters have also been developed (27). An advantage of reporter gene imaging is the ability to develop and validate indirect-imaging 


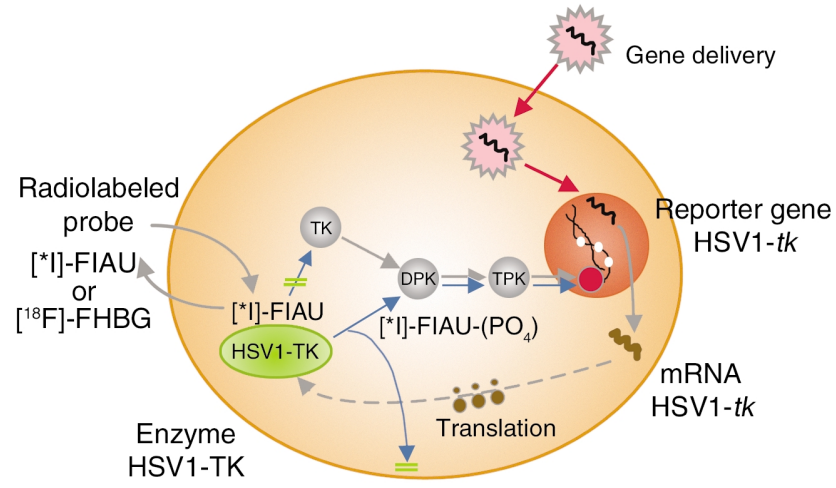

Figure 1

Schematic for imaging herpes simplex virus 1 thymidine kinase reporter gene (HSV1-tk) expression with reporter probes ${ }^{124}$ I- or ${ }^{131}$ I-labeled FIAU and ${ }^{18} \mathrm{~F}$-labeled FHBG. The HSV1-tk gene complex is transfected into target cells by a vector. Inside the transfected cell, the HSV1-tk gene is transcribed to HSV1-tk mRNA and then translated on the ribosomes to a protein (enzyme), HSV1-TK. After administration of a radiolabeled probe and its transport into the cell, the probe is phosphorylated by HSV1-TK (gene product). The phosphorylated radiolabeled probe does not readily cross the cell membrane and is trapped within the cell. Thus, the magnitude of probe accumulation in the cell (i.e., the level of radioactivity) reflects the level of HSV1-TK enzyme activity and the level of HSV1-tk gene expression.

strategies more rapidly and at considerably lower cost than direct-imaging strategies. This is possible because only a small number of well-characterized and validated reporter gene-reporter probe pairs need to be established for noninvasive reporter gene imaging. This is because a single reporter gene-reporter probe pair combination can be used in many different reporter constructs to image many different biological and molecular-genetic processes. Indirect molecular imaging is already providing the opportunity for a wider application of imaging in the study of experimental animal models of human disease, and for their implementation in future clinical studies.

\section{Reporter gene imaging}

A common feature of all reporter vectors is the cDNA expression cassette containing the reporter transgene(s) of interest (e.g., HSV1-tk). The advantage of reporter vectors is that the design and arrangement of the expression cassette can be varied. For example, the reporter transgene(s) can be driven by any promoter/enhancer sequence of choice. The promoter can be constitutive (leading to continuous transcription), or it can be inducible (leading to controlled expression). The promoter can also be cell specific, allowing expression of the transgene to be restricted to certain cells and organs. The paradigm for quantitative imaging of transgene expression involves several steps, including the initiation of transcription (that can be controlled by specific promoter/ enhancer elements), the process of DNA transcription and stabilization of mRNA, and subsequent translation of mRNA into the gene product. In this manner, the reporter expression cassette can be designed to provide information about endogenous gene regulation, mRNA stabilization, and specific protein-protein interactions.

A general paradigm for gene imaging using radiolabeled probes was initially described in 1995 (7) (Figure $1)$. It is important to note that imaging transgene expression is independent of the vector used to transfect and transduce target tissue. Any of several currently available vectors can be used (e.g., retrovirus, adenovirus, adenoassociated virus, lentivirus, liposomes, etc.). The reporter transgene can encode for an enzyme (e.g., HSV1-tk), a receptor (e.g., the human dopamine 2 receptor, $\mathrm{h} D 2 R$, and the human somatostatin receptor subtype 2, hSSTR2), or a transporter (e.g., the human sodium iodide symporter, hNIS).

Wild-type HSV1-tk (9) and a mutant HSV1-tk gene, HSV1-sr39tk (28), are the reporter genes most commonly used in current molecular imaging studies that use radiolabeled probes and PET. The HSV1-tk and HSV1-sr39tk gene products are proteins (enzymes) that have less substrate specificity than mammalian thymidine kinase 1 (TK1). The viral kinases phosphorylate a wider range of compounds, including acycloguanosines (e.g., acyclovir, ganciclovir, and 9-[4-fluoro-3-(hydoxymethyl)butyl]guanine [FHBG] ) and $2^{\prime}$-fluoro-nucleoside analogues of thymidine (e.g., 5-iodo-2'-fluoro- 2 'deoxy-1- $\beta$-D-arabino-furanosyl-uracil [FIAU]). This difference between mammalian and viral TK enzymes permits the development and use of radiolabeled probes that are phosphorylated to a significantly greater extent by HSV1-TK or HSV1-sr39TK in comparison to mammalian TK1. These viral gene products selectively metabolize their complementary radiolabeled probes, which results in probe entrapment and accumulation in transduced cells. A paired comparison of the efficacy of different radiolabeled probes in imaging of HSV1-tk expression has been reported recently (29).

A reporter gene can also encode for an extracellular or intracellular receptor or a cell membrane transporter (e.g., $\mathrm{h} D 2 R, \mathrm{~h} S S T R 2$, or $\mathrm{hNIS}$ ) that binds or transports a radiolabeled or paramagnetic probe. The human genes $\mathrm{h} D 2 R(30)$, hSSTR2 (31), and hNIS (18) have been suggested as potential reporter genes for human studies. All three of these genes have limited expression in the body: $\mathrm{h} D 2 R$ expression is limited to the striatalnigral system of the brain, high hSSTR2 expression is limited largely to carcinoid tumors, and hNIS expression is limited largely to thyroid, stomach, and salivary glands. This is a very clever strategy, because the reporter gene products are less likely to be immunogenic, and complementary radiolabeled probes are established for each of these reporter genes that are approved for human administration: $3-\left(2^{\prime}-\left[{ }^{18} \mathrm{~F}\right]\right.$ fluoroethyl)spiperone for $\mathrm{h} D 2 R$ imaging (32), [11 $\mathrm{In}]$ diethylenetriamine pentaacetic acid-octreotide (a complementary radiolabeled somatostatin analogue) for hSSTR2 imaging (33), and radiolabeled iodide or 
pertechnetate for hNIS imaging $(18,34)$. These three reporter systems have distinct benefits and are good candidates with respect to regulatory approval for molecular/reporter imaging in human subjects. However, receptor and transporter expression on the surface of cells is a complex process and involves intracellular trafficking and cell membrane incorporation that could be altered under different conditions and different disease states. It remains to be shown whether imaging receptor- and transporter-based reporters (e.g., the $\mathrm{h} D 2 R, \mathrm{~h} S S T R 2$, and $\mathrm{hNIS}$ systems) will provide a consistent and reliable measure of reporter gene expression under variable stress conditions.

\section{Surrogate imaging}

"Surrogate marker" probes that reflect the downstream effects of one or more endogenous moleculargenetic processes are also being explored. This approach is particularly attractive for potential translation into clinical studies in the near term, since direct and indirect molecular imaging studies are only beginning to be implemented in the clinic (35). Existing radiopharmaceuticals and imaging paradigms may be useful for monitoring downstream effects of changes in specific molecular-genetic pathways in diseases such as cancer. However, the surrogate-imaging paradigms are likely to be less specific and more limited with respect to the number of molecular-genetic processes that can be imaged. Nevertheless, surrogate imaging benefits from the use of probes that have already been developed and studied in human subjects. The translation of surrogate-marker imaging into treatment-assessment paradigms of patients will be far easier than translation of the reporter-transgene or the direct-imaging paradigms outlined above. Nevertheless, it remains to be shown whether there is a sufficiently high correlation between surrogate-marker imaging and direct molecular assays that reflect the activity of a particular molecular-genetic pathway of interest.

The application of surrogate imaging to monitoring of treatment response is gaining increasing attention, particularly as it relates to the development and testing of new pathway-specific drugs. For example, the assessment of noncytotoxic, cytostatic drugs such as the antiangiogenic class of drugs poses particular problems for imaging assessments based on tumor volume. Surrogate-imaging markers of tumor vascularity are being proposed for assessment of antiangiogenesis treatment response. One recent example of clinically useful surrogate imaging for early assessment of treatment response is FDG PET imaging of gastrointestinal stromal tumors (GISTs) before and after STI571 (Gleevec) treatment (Figure 2) $(36,37)$. Although glucose uptake and glycolytic enzyme activity are homeostatically regulated, glucose metabolism has been to shown to be regulated, in some instances, by extracellular signals mediated by cell surface receptors. These instances include the
CD28 signaling pathway in T cells and insulin receptor signaling (38). Receptor-mediated regulation of glucose uptake is thought to involve activation of PI3K, Akt, mTOR, and S6 kinase. A likely explanation for the dramatic effect of STI571 (Gleevec) on FDG uptake in GISTs is that Kit receptor signaling regulates glucose uptake as well as glucose metabolism. Interestingly, in yeast and a lymphocyte cell line, mTOR depletion or rapamycin treatment and glucose deprivation trigger a stress response similar to a starvation phenotype (39). Whether imaging of surrogate markers will be valuable for assessment of treatment directed at other molecular-genetic abnormalities in tumors (EGFR, p53, c-Met, hypoxiainducible factor 1 , etc.) remains to be demonstrated.

\section{Applications of PET- and optical-based reporter gene imaging: monitoring gene therapy and therapeutic gene imaging}

A noninvasive, clinically applicable method of imaging the expression of successful gene transduction in target tissue or specific organs of the body would be of considerable value for monitoring and evaluating gene therapy in human subjects $(35,40)$. PET imaging could define the location, magnitude, and persistence of gene expression over time. Targeting of gene therapy to a particular tissue (e.g., tumor) or specific organs of the body is an increasingly active area of research. Several important issues for clinical optimization of gene therapy remain unresolved in many current clinical protocols: (a) Has gene transduction or transfection been successful? (b) Is the distribution of the transduced or transfected gene localized to the target organ or to target tissue, and is the distribution in the target optimal? (c) Is the level of transgene expression in the target organ or tissue sufficient to result in a therapeutic effect? (d) Does the transduced or transfected gene localize to any organ or tissue at sufficient levels to induce unwanted toxicity? (e) In the case of combined prodrug and gene therapy protocols, when is transgene expression at a maximum, and when is the optimal time to initiate treatment with the prodrug? (f) How long does transgene expression persist in the target and other tissues?

Noninvasive-imaging techniques using selected reporter gene and reporter probe combinations will provide a practical and clinically useful way to identify successful gene transduction and expression in patients undergoing gene therapy. One could argue that biopsies of target tissue or blood sampling and analysis of transgene expression, or assay for secretable marker (e.g., carcinoembryonic antigen) could be performed and that imaging is not critical. However, imaging provides some clear advantages, including (a) the ability to repeatedly assess gene expression over time, especially when multiple sequential biopsies are not feasible; (b) the absence of any perturbation of the underlying tissue, which occurs with biopsy 

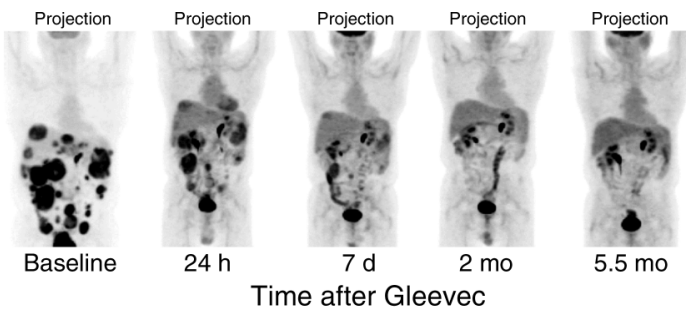

Figure 2

FDG positron emission tomography (PET) imaging before and after treatment. In patients with gastrointestinal stromal tumors (GIST), tumor glucose utilization is very high and these tumors can be readily visualizd by $[18 \mathrm{~F}]^{-}$fluorodeoxyglucose (FDG) PET $(36,37)$. A striking observation in patients with GIST who are treated with STI571 (Gleevec) was that the rapid and sustained decrease in fluorodeoxglucose (FDG) uptake determined by PET scan was seen as early as 24 hours after one dose of ST1571, and this was sustained over many months. The high FDG levels seen in the kidney, ureter, and bladder are normal in both the pre- and post-Gleevec images. Figure adapted from ref. 37, with appreciation and permission from Annick Van en Abbeele, Danaa-Farber Cancer Institute, Boston, Massachusetts, USA.

procedures; and (c) the ability to obtain 3D spatial information in the entire body as well as target organs and tumors, which could be of considerable value in addressing toxicity issues.

HSV1-tk has the advantage of being both a therapeutic gene (combined with ganciclovir treatment) and a reporter gene (using an appropriate radiolabeled probe, such as FIAU or FHBG). This combination allows for direct imaging of the therapeutic gene product (HSV1-TK) and can be used to define the location, magnitude, and duration of HSV1-tk gene expression. Experimental validation of this approach has been demonstrated in animal models of colorectal metastases to the liver treated either with adenovirus-mediated HSV1-tk gene transfer and ganciclovir (suicide gene therapy) (41-43) or with conditionally replicating, oncolytic herpes viruses that constitutively express the HSV1-tk gene $(44,45)$. However, most therapeutic genes do not lend themselves to direct imaging of their transgene product. Furthermore, the development and validation of a new probe and a new imaging paradigm specific to each therapeutic transgene of interest would be a very costly and time-consuming endeavor. Many therapeutic gene products do not readily lend themselves to radionuclide assessment, and it may not even be possible in some cases. It is therefore more reasonable to consider alternative strategies for indirect imaging of therapeutic gene expression that use established reporter gene-reporter probe combinations.

\section{Imaging endogenous biological processes}

Indirect-imaging strategies using reporter gene technology have been employed to visualize transcriptional and posttranscriptional regulation of target-gene expression, as well as specific intracellular proteinprotein interactions. Several examples are provided below. Imaging the transcriptional regulation of endogenous genes in living animals (and potentially in human subjects) using noninvasive-imaging techniques can provide a better understanding of normal and cancer-related biological processes. A recent paper by our group was the first to show that $\mathrm{p} 53$-dependent gene expression can be imaged in vivo with PET and by in situ fluorescence (25). A retroviral vector (Cis-p53/TKeGFP) was generated by placement of HSV1-tk and enhanced GFP fusion gene (TKeGFP, a dual-reporter gene) under control of a p53-specific response element. DNA damage-induced upregulation of p53 transcriptional activity was demonstrated and correlated with the expression of p53-dependent downstream genes (including p21). These findings were observed in $\mathrm{p} 53^{+/+}$cells and xenografts, but not in $\mathrm{p} 53^{-/-}$cells. This was the first demonstration that a Cis reporter system (Cis-p53/TKGFP) was sufficiently sensitive to image endogenous gene expression using noninvasive nuclear (PET) imaging (Figure 3, a-e). The PET images corresponded with upregulation of genes in the p53 signal transduction pathway (p53dependent genes) in response to DNA damage induced by $\mathrm{N}, \mathrm{N}^{\prime}$-bis(2 chloroethyl)-N-nitrosourea (BCNU) chemotherapy (Figure 3, f-m). PET imaging of p53 transcriptional activity in tumors using the Cis-p53/TKGFP reporter system could be used to assess the effects of new drugs or other novel therapeutic paradigms that are mediated through $\mathrm{p} 53$ dependent pathways. For example, specific p53 gene therapy strategies that are based on p53 overexpression (46) could be monitored by noninvasive imaging.

Imaging of endogenous gene expression may be hampered when weak promoters, in their usual Cis configuration, are activated. This results in weak transcriptional activity of the reporter gene. In one study, enhancement of the transcriptional activity of the androgen-responsive prostate-specific antigen enhancer (PSE) was achieved using a two-step transcriptional amplification (TSTA) approach to amplify expression of firefly luciferase and mutant HSV1-tk (HSV1-sr39tk) in a prostate cancer cell line (LNCaP) (27). The androgen-responsive TSTA system for reporter gene expression was improved further using a chimeric TSTA system that employs duplicated variants of the prostate-specific antigen (PSA) gene enhancer to express GAL4 derivatives fused to one, two, or four VP16 activation domains. A very encouraging result was the demonstration that the TSTA system was androgen concentration sensitive, suggesting a continuous rather than a binary reporter response. In another study, methods to enhance the transcriptional activity of the carcinoembryonic antigen (CEA) promoter using a trans system (similar to the TSTA described above) were employed to selectively treat and image CEA-expressing tumors (41). To increase promoter strength while maintaining tissue specificity, a recombinant adenovirus was constructed that contained a TSTA system with a tumor- 
specific CEA promoter driving a transcription transactivator, which then activates a minimal promoter to drive expression of the HSV1-tk suicide/reporter gene. This adenovirus/CEA-binary-tk system resulted in equal or greater killing of transduced cells by ganciclovir in a CEA-specific manner, compared with killing by ganciclovir of cells transduced with a CEAindependent vector containing a constitutive viral promoter driving HSV-tk expression (adenovirus/ RSV-tk). However, as observed with the PSE-TSTA reporter system above, the in vivo imaging comparison of the TSTA and Cis reporter systems showed substantially less dramatic differences than were obtained by the in vitro analyses.

Gene expression levels are also regulated by posttranscriptional modulation, including the translation of mRNA. A recent study demonstrated that imaging of posttranscriptional regulation of gene expression is feasible. Cells were exposed to antifolates, and a rapid increase in the level of the enzyme dihydrofolate reductase (DHFR) was induced. Several studies indicated that the DHFR enzyme binds to its own mRNA in the coding region, and that inhibition of DHFR by methotrexate releases the DHFR enzyme from the mRNA. Consequently, this release results in an increase in translation of DHFR protein (47). In addition to the described translational regulation of DHFR in cancer cells exposed to methotrexate, increased levels of DHFR also can occur by DHFR gene amplification, a common mechanism of acquired resistance to this drug. In contrast to rapid translational modulation of DHFR, gene amplification occurs in response to chronic exposure to antifolates, and elevated cellular levels of DHFR result from transcription of multiple DHFR gene copies. Recently, we showed that this adaptive cellular response mechanism could be used to determine whether posttranscriptional regulation of gene expression could be a

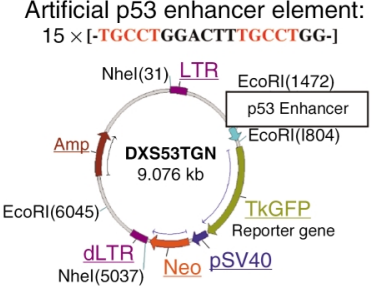

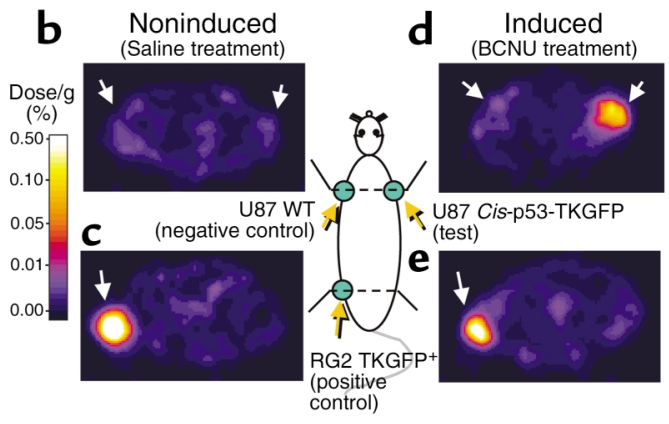

RT-PCR
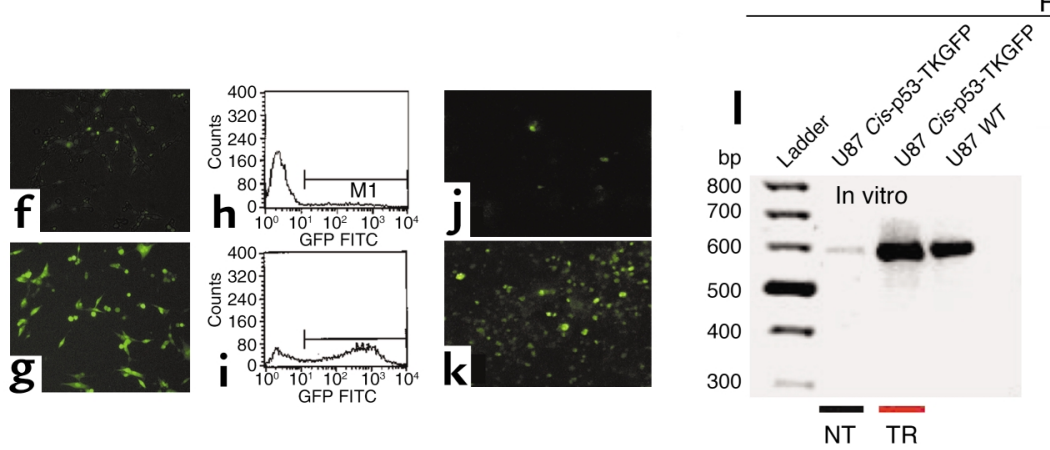

$\mathbf{m}$

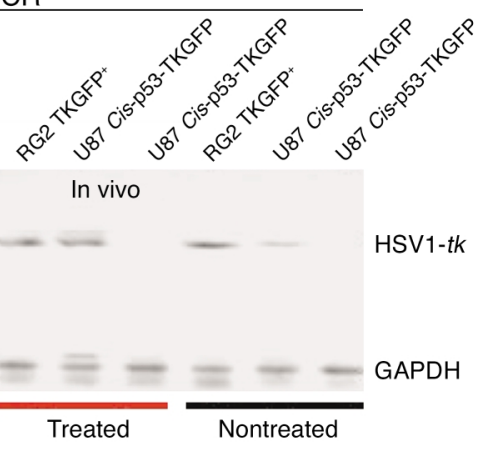

Figure 3

PET imaging of endogenous p53 activation and validation of Cis-p53/TKGFP reporter system in cell cultures and sampled tumor tissue. (a) The p53-sensitive reporter vector contains an artificial p53-specific enhancer that activates expression of the TKeGFP reporter gene. (b-e) Transaxial PET images (GE Advance tomograph; General Electric Co., Milwaukee, Wisconsin, USA) through the shoulder (b and $\mathbf{d}$ ) and pelvis ( $\mathbf{c}$ and $\mathbf{e}$ ) of two rats are shown (upper panel); the images are color-coded to the same radioactivity scale (percent dose/g). An untreated animal is shown on the left ( $\mathbf{b}$ and $\mathbf{c}$ ), and a BCNU-treated animal is shown on the right ( $\mathbf{d}$ and $\mathbf{e})$. Both animals have three subcutaneous tumor xenografts: U87 Cis-p53-TKGFP (test) in the right shoulder, U87 wild type (negative control) in the left shoulder, and RG2 TKGFP+ (positive control) in the left thigh. The untreated animal on the left shows localization of radioactivity only in the positive control tumor $\left(\mathrm{RG}_{2} \mathrm{TKGFP}^{+}, \mathbf{c}\right)$; the test (U87 Cis-p53-TKGFP, b) and negative control (U87wt, b) tumors are at background levels. The BCNU-treated ani$\mathrm{mal}$ on the right shows significant radioactivity localization in the test tumor (right shoulder, $\mathbf{d}$ ) and in the positive control (left thigh, $\mathbf{e}$ ), but no radioactivity above background in the negative control (left shoulder, $\mathbf{d})$. ( $\mathbf{f}-\mathbf{i})$ Fluorescence microscopy and FACS analysis of a transduced U87 Cis-p53-TKGFP cell population in the noninduced (control) state ( $\mathbf{f}$ and $\mathbf{h}$ ), and 24 hours after a 2 -hour treatment with $40 \mu \mathrm{g} / \mathrm{ml}$ BCNU ( $\mathbf{g}$ and $\mathbf{i}$ ). ( $\mathbf{j}$ and $\mathbf{k}$ ) Fluorescence microscopic images of U87 Cis-p53-TKGFP subcutaneous tumor samples obtained from untreated rats (j) and rats treated intraperitoneally with $40 \mathrm{mg} / \mathrm{kg} \mathrm{BCNU}(\mathbf{k})$. The RT-PCR blots from in vitro (I) and in vivo (m) experiments show very low HSV1-tk expression in untreated U87 Cis-p53-TKGFP transduced cells and xenograft-bearing animals, respectively, and no HSV1-tk expression in wild-type U87 cells and tumor tissue, respectively. When U87 Cis-p53-TKGFP transduced cells and xenograft-bearing animals are treated with BCNU, there is a marked increase in HSV1-tk expression comparable to that in constitutively HSV1-tk-expressing RG2TK ${ }^{+}$ cells and xenografts. Adapted with permission from ref. 25. 

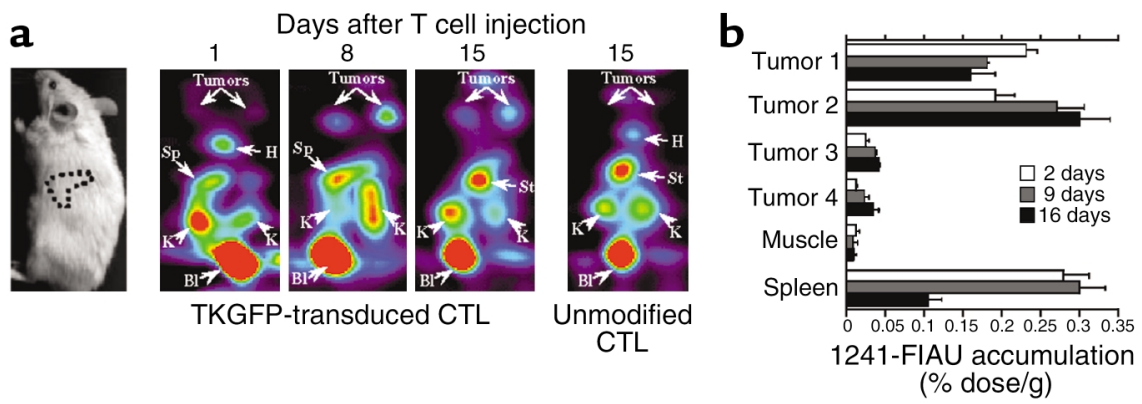

\begin{abstract}
Figure 4
Imaging T cell migration and targeting. (a) Sequential oblique projections of summed coronal MicroPET images of a mouse bearing autologous Epstein-Barr virus-transformed B-cell lines (BLCL) (tumor 1), HLA-A0201-matched BLCL (tumor 2), HLA-mismatched BLCL (tumor 3), and HLA-A0201 ALL (tumor 4) tumors located in the left and right shoulders and the left and right thighs, respectively. All images are from a single representative animal at a $45^{\circ}$ angle from the prone position to visualize the spleen, and all images were obtained 4 hours after [124I]FIAU injection on days 1, 8, and 15 after T cell infusion. K, kidneys; $\mathrm{H}$, heart; St, stomach; Sp, spleen; Bl, bladder. (b) Graph of radioactivity levels (percent dose/g) that localized in different tumors and tissues, at three time points (2, 9, and 16 days) after EBV-specific TKGFP-CTL administration. Radioactivity was measured 24 hours after [124/]FIAU injection. Adapted with permission from ref. 57.
\end{abstract}

monitored by reporter-PET imaging (48). The results of this study indicated that the increase in reporter protein and enzyme (DHFR-HSV1-TK) activity was occurring at a translational level, rather than at the transcriptional level. This effect could be visualized by [124I]FIAU and PET imaging studies that were performed on nude rats bearing DHFR-HSV1-TK-transduced HCT-8 xenografts.

\section{Imaging protein-protein interactions}

Two recent studies have shown that it is possible to image protein-protein interactions $(49,50)$. The interaction of two proteins, ID and MyoD, was the focus of the first study. To modulate the expression of these two proteins, the NF- $\mathrm{KB}$ promoter was used to drive expression of the id-gal4 and/or myoD-vp16 fusion genes, and TNF- $\alpha$ was used to induce the NF- $\kappa B$ promoter (49). The reporter construct contained five GAL4 DNAbinding sites driving the expression of the firefly luciferase $(f l)$ reporter gene. Eight hours after TNF- $\alpha$ administration, the mice showed a significantly greater level of $f l$ expression ( 20 -fold at 8 hours, fivefold at 20 hours, and threefold at 30 hours) compared with mice that did not receive TNF- $\alpha$, and an approximately 60 fold greater level compared with mice injected with $293 \mathrm{~T}$ cells transfected with noninteracting protein partners (MyoD and p53). In the second study, proteinprotein interactions could be imaged in vivo with PET and by fluorescence imaging using a variant of the HSV1-tk/GFP dual-reporter fusion gene (50). It is well known that TAg, which is the transforming protein of SV40 virus, binds constitutively to p53 and blocks transactivation of p53 target genes, leading to transformation of mammalian cells. In this study, the authors used an approach similar to that described above (49), but they used a different reporter system involving a reverse tetracycline-responsive transactivator expressed from a cytomegalovirus promoter and a plasmid expressing either GAL4-BD-p53 and VP16TAg or GAL4-BD-p53 and VP16-CP from a bidirec- tional, tetracycline-regulated promoter. Mice bearing the test and control transduced xenografts were treated with doxycycline for 48 hours to induce expression of the hybrid proteins. Visualization of the GAL4mNLS-sr39TK-eGFP reporter at 48 hours was accomplished with $\left[{ }^{18} \mathrm{~F}\right] \mathrm{FHBG}$ and micro-PET imaging. Based on region-of-interest values from the micro-PET images, the uptake of $\left[{ }^{18} \mathrm{~F}\right] \mathrm{FHBG}$ was 5.5 -fold greater in TAg (test) xenografts than in control xenografts.

These initial results are very encouraging, but they are also limited, since they demonstrate only constitutive interactions of the known pairs of interacting proteins. Further studies will be required to validate the approach with respect to the sensitivity and dynamic range of these reporter systems for monitoring the induction and inhibition of endogenous protein-protein interactions.

\section{Imaging adoptive therapy}

Reporter gene imaging is also being used to visualize adoptively transferred cells (e.g., T lymphocytes and stem/progenitor cells). Genetically labeled cells have been used previously for monitoring adoptive therapy; this dates back to the late 1980s (51-56). Although reporter gene expression could be detected by PCR or FACS analysis of peripheral blood, the in vivo distribution of infused cells in the body over time cannot be addressed by this approach. The longterm trafficking and localization of $\mathrm{T}$ lymphocytes play an important role in the immune response and in the elimination of abnormal cells and infectious agents from the body.

Ongoing studies in our laboratory aim to image the in vivo targeting and the accumulation of EBV lymphoma-specific cytotoxic $\mathrm{T}$ cells in autologous and allogeneic HLA-matched $\mathrm{EBV}^{+} \mathrm{B}$ lymphomas using PET and optical imaging modalities (57). CTLs specific for homogenous EBV-transformed B lymphocytes were obtained and stably transduced with a constitutively expressing dual-reporter gene (HSV1-tk/eGFP 
fusion gene). Specific accumulation and localization of radioactivity were observed only in the autologous and allogeneic HLA-matched $\mathrm{EBV}^{+} \mathrm{B}$ lymphoma and in the spleen; no localization was seen in the allogeneic HLA-matched EBV- lymphoma and the HLA-mismatched $\mathrm{EBV}^{+}$lymphoma (Figure 4). Sequential imaging over 72 hours in another set of animals showed trafficking as well as targeting of the transduced and radiolabeled CTLs. These studies indicate that it may be feasible to isolate and transduce CTLs (and other immune-specific cells or stem/progenitor cells) with reporter constructs and then to monitor their targeting and proliferation in the donor or HLA-matched recipient using noninvasive reporter gene PET imaging. Importantly, these studies can be repeated in the same subject sequentially over time, because the adoptively transferred CTLs have been genetically labeled with a reporter gene.

When combined with the imaging of $T$ cell activation mediated by nuclear factor of activated $\mathrm{T}$ cells (NFAT) (26), noninvasive imaging using PET would allow for monitoring of the trafficking, proliferation, and antigen-specific activation of $\mathrm{T}$ cells in antitumor vaccination trials (Figure 5). A double-reporter system (containing a constitutive reporter to identify the site, number, and duration of $\mathrm{T}$ cell location, plus an inducible reporter to identify the site, intensity, and duration of T cell activation, e.g., NFAT expression) could be used for genetic labeling of $\mathrm{T}$ cells prior to adoptive therapy. This double-reporter system could address several important questions related to adoptive $\mathrm{T}$ cell therapies. For example, is there substantial proliferation of adoptively transferred $\mathrm{T}$ cells at the target site, or do activation and proliferation occur at other sites (e.g., specific lymphoid organ sites), followed by migration and localization to the target site? This question could potentially be addressed quantitatively by repetitive PET imaging of the doublereporter system described above, and repetitive imaging of the same animal or subject over time.

\footnotetext{
Figure 5

Imaging NFAT-TKGFP reporter system activity with PET and optical fluorescence, plus assessments in tissue samples. (a) Photographic image of a typical mouse bearing different subcutaneous infiltrates (middle panel). Transaxial PET images (GE Advance tomography) of TKGFP expression in a mouse treated with control antibody (left panel) or anti-CD3/CD28 antibodies (right panel) were obtained at the levels indicated by the dashed lines. (b) [ $\left.{ }^{124} \mathrm{I}\right] \mathrm{FIAU}$ accumulation (percent dose/g) in tissue samples of the Jurkat/dcmNFATtgn transduced infiltrates produced from clone 3 and 4 cell lines, wild-type Jurkat infiltrates, and blood plasma, obtained after PET imaging. (c) FACS profiles of TKGFP and CD69 expression in a tissue sample from the same Jurkat/dcmNFATtgn clone 4 infiltrate that was imaged with PET. (d-f) Images ofTKGFP fluorescence in a subcutaneous Jurkat/dcmNFATtgn (clone 4) infiltrate were obtained in the same animal (d), first before (e) and then after (f) treatment with anti-CD3/CD28 antibodies. Figure adapted with permission from ref. 26.
}

\section{Issues for the future}

Future success depends on bringing the imaging disciplines closer together - as we have seen in the complementary benefits of dual-modality reporters incorporating both optical and nuclear imaging technology - as well as on developing closer and more interactive relationships between the imaging community and our molecular biology and cell biology colleagues. Complementary instrumentation for imaging both animals and patients provides a unique opportunity for substantive translational research. Animals bearing transduced xenografts that contain specific reporter constructs and animals transduced with viral vectors that bear these reporter constructs currently provide the main resource for further advances in in vivo molecular imaging research. Transgenic animals that express reporter constructs in a system-wide manner, or selectively in certain organs or cell types in the adult organism or during a particular phase of embryonic development, are the subject of active current research. Noninvasive imaging can assist in these studies by assessing the activation or suppression of specific endogenous genes, and the activity of specific signal transduction pathways and specific protein-protein interactions. This information could be useful in defining and monitoring

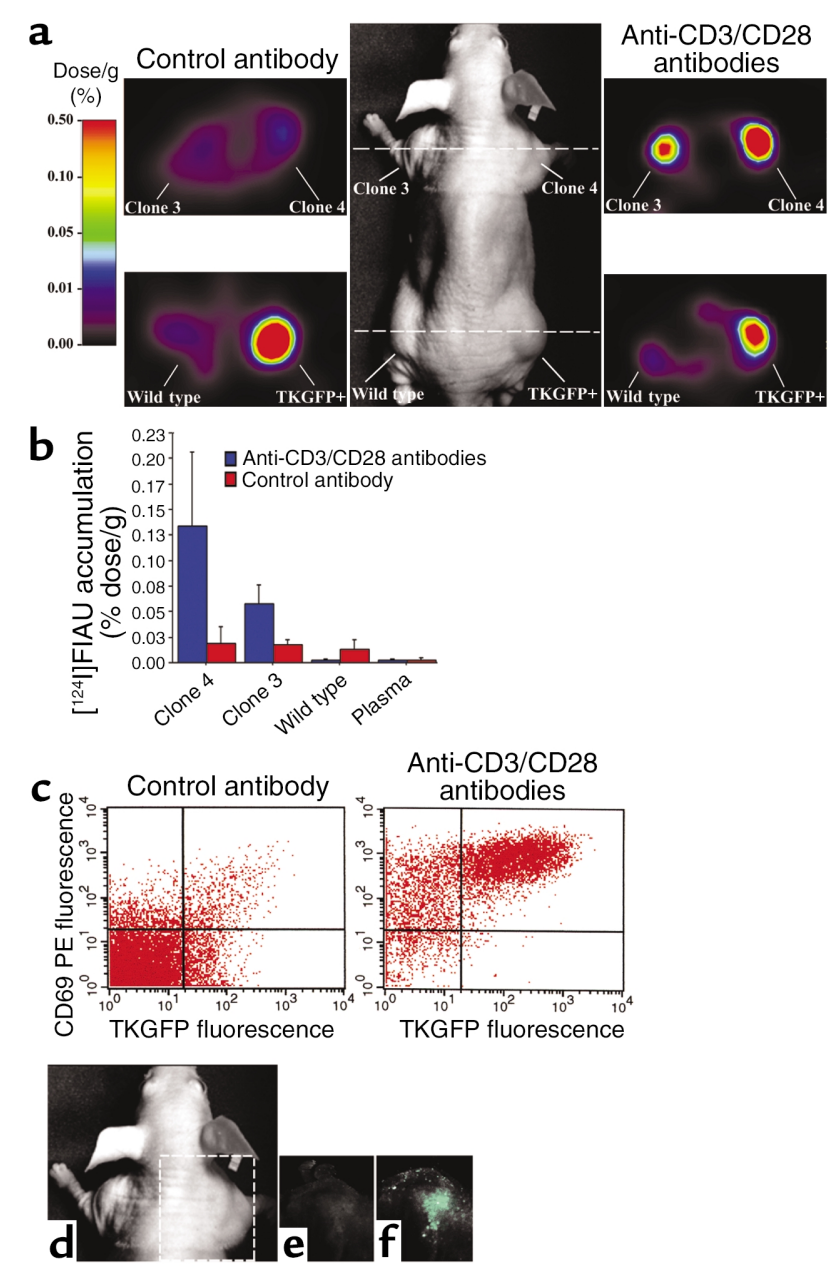


cell transformation to a more malignant state, or determining whether a specific drug effectively targets a specific signal transduction pathway.

Reporter gene imaging studies will be more limited in patients than in animals, because of the necessity of transducing target tissue with specific reporter constructs. Ideal vectors to target specific organs or tissues (tumors) do not exist at this time, although this is an active area of human gene therapy research. Each new vector requires extensive and time-consuming safety testing before regulatory approval for human administration. Similarly, radiolabeled probes for existing and new reporter systems, and of the probes being developed for new direct-imaging paradigms, will require safety testing and regulatory approval. The translation of molecular imaging research from animal proof-of-principle experiments into patient studies and the subsequent clinical application of these imaging paradigms will develop more slowly under careful monitoring.

The primary applications of noninvasive in vivo marker/reporter gene imaging are likely to be (a) quantitative monitoring of transduction efficacy in gene therapy and animal research protocols by imaging of the location, extent, and duration of transgene expression; (b) monitoring of cell trafficking, targeting, and replication in adoptive therapies, involving ex vivo transduction of harvested immune-competent cells and stem/progenitor cells; (c) development of a library of transgenic reporter mice that can be cross-bred to create double-, triple-, or multipletransgenic animal colonies - specifically, animal models of human disease with the capability for repetitive-longitudinal noninvasive reporter imaging; and (d) assessment of endogenous molecular events using different reporter gene imaging technologies. However, these advances and translation into patient studies requires the development of safe, efficient, and target-specific vectors for diagnostic transductions and reporter imaging.

We remain optimistic; the initial noninvasive reporter gene imaging study in patients involved the monitoring of HSV1-tk-ganciclovir gene therapy of patients with glioblastoma (58), and additional gene imaging studies in patients are likely to be published in the near future. The advantages and benefits of noninvasive imaging to monitor transgene expression in gene therapy protocols are clear. The ability to visualize transcriptional and posttranscriptional regulation of endogenous target-gene expression, as well as specific intracellular protein-protein interactions in patients, will provide the opportunity for new experimental venues in patients. Hopefully these will include imaging the malignant phenotype of an individual patient's tumor at a molecular level and monitoring changes in tumor phenotype over time. The potential to image a drug's effect on a specific signal transduction pathway in an individual patient's tumor provides the opportunity to monitor treatment response at the molecular level. At the moment this requires the use of diagnostic reporter gene transduction vectors that target specific organs or tissues (tumors), and this will initially limit the translation and application of reporter gene technology to patients. However, direct and surrogate molecular imaging may begin to fill this gap over the next decade.

1. Blasberg, R., and Gelovani Tjuvajev, J. 2002. Molecular imaging: a nuclear based perspective. Molecular Imaging. 1:160-180.

2. Weissleder, R., Reimer, P., Lee, A.S., Wittenberg, J., and Brady, T.J. 1990. MR receptor imaging: ultra small iron oxide particles targeted to asialoglycoprotein receptors. Am. J. Roentgenol. 6:1161-1167.

3. Weissleder, R., et al. 1991. Polyclonal human immunoglobulin G labeled with polymeric iron oxide: antibody MR imaging. Radiology. 1:245-249.

4. Kayyem, J.F., Kumar, R.M., Fraser, S.E., and Meade, T.J. 1995. Receptortargeted co-transport of DNA and magnetic resonance contrast agents. Chem. Biol. 9:615-620.

5. Weissleder, R., et al. 1997. MR imaging and scintigraphy of gene expression through melanin induction. Radiology. 2:425-429.

6. Louie, A.Y., et al. 2000. In vivo visualization of gene expression using magnetic resonance imaging. Nat. Biotechnol. 3:321-325.

7. Tjuvajev, J.G., et al. 1995. Imaging the expression of transfected genes in vivo. Cancer Res. 55:6126-6132.

8. Tjuvajev, J.G., et al. 1996. Noninvasive imaging of herpes virus thymidine kinase gene transfer and expression: a potential method for monitoring clinical gene therapy. Cancer Res. 56:4087-4095.

9. Tjuvajev, J.G., et al. 1998. Imaging herpes virus thymidine kinase gene transfer and expression by positron emission tomography. Cancer Res. 58:4333-4341.

10. Gambhir, S.S., et al. 1998. Imaging of adenoviral-directed herpes simplex virus type 1 thymidine kinase reporter gene expression in mice with radiolabeled ganciclovir. J. Nucl. Med. 11:2003-2011.

11. Gambhir, S.S., et al. 1999. Imaging adenoviral-directed reporter gene expression in living animals with positron emission tomography. Proc. Natl. Acad. Sci. U. S. A. 5:2333-2338.

12. Contag, C.H., et al. 1997. Visualizing gene expression in living mammals using a bioluminescent reporter. Photochem. Photobiol. 66:523-531.

13. Contag, P.R., Olomu, I.N., Stevenson, D.K., and Contag, C.H. 1998. Bioluminescent indicators in living mammals. Nat. Med. 4:245-247.

14. Rehemtulla, A., et al. 2000. Rapid and quantitative assessment of cancer treatment response using in vivo bioluminescence imaging. Neoplasia. 6:491-495.

15. Sokoloff, L., et al. 1977. The $\left[{ }^{14} \mathrm{C}\right]$ deoxyglucose method for the measurement of local cerebral glucose utilization: theory, procedure, and normal values in the conscious and anesthetized albino rat. J. Neurochem. 5:897-916

16. Reivich, M., et al. 1977. Measurement of local cerebral glucose metabolism in man with 18F-2-fluoro-2-deoxy-d-glucose. Acta Neurol. Scand. Suppl. 64:190-191.

17. Miyagawa, T., et al. 1998. "Facilitated" amino acid transport is up-regulated in brain tumors. J. Cereb. Blood Flow Metab. 18:500-509.

18. Haberkorn, U. 2001. Gene therapy with sodium/iodide symporter in hepatocarcinoma. Exp. Clin. Endocrinol. Diabetes. 1:60-62.

19. Dewanjee, M.K., et al. 1994. Noninvasive imaging of c-myc oncogene messenger RNA with indium-111-antisense probes in a mammary tumor-bearing mouse model. J. Nucl. Med. 6:1054-1063.

20. Tavitian, B., et al. 1998. In vivo imaging of oligonucleotides with positron emission tomography. Nat. Med. 4:467-471.

21. Tjuvajev, J.G., et al. 1999. A general approach to the non-invasive imaging of transgenes using cis-linked herpes simplex virus thymidine kinase. Neoplasia. 4:315-320.

22. Yu, Y., et al. 2000. Quantification of target gene expression by imaging reporter gene expression in living animals. Nat. Med. 8:933-937.

23. Sweeney, T.J., et al. 1999. Visualizing the kinetics of tumor-cell clearance in living animals. Proc. Natl. Acad. Sci. U. S. A. 21:12044-12049.

24. Mayerhofer, R., Araki, K., and Szalay, A.A. 1995. Monitoring of spatial expression of firefly luciferase in transformed zebrafish. J. Biolumin. Chemilumin. 5:271-275.

25. Doubrovin, M., et al. 2001. Imaging transcriptional regulation of p53dependent genes with positron emission tomography in vivo. Proc. Natl. Acad. Sci. U. S. A. 98:9300-9305.

26. Ponomarev, V., et al. 2001. Imaging TCR-dependent NFAT-mediated T-cell activation with positron emission tomography in vivo. Neoplasia. 3:480-488.

27. Iyer, M., et al. 2001. Two-step transcriptional amplification as a method for imaging reporter gene expression using weak promoters. Proc. Natl. Acad. Sci. U. S. A. 25:14595-14600.

28. Gambhir, S.S., et al. 2000. A mutant herpes simplex virus type 1 thymi- 
dine kinase reporter gene shows improved sensitivity for imaging reporter gene expression with positron emission tomography. Proc. Natl. Acad. Sci.U. S. A. 6:2785-2790.

29. Tjuvajev, J.G., et al. 2002. Comparison of radiolabeled nucleoside probes (FIAU, FHBG and FHPG) for PET imaging of HSV1-tk gene expression. J. Nucl. Med. 8:1072-1083.

30. MacLaren, D.C., et al. 1999. Repetitive, non-invasive imaging of the dopamine D2 receptor as a reporter gene in living animals. Gene Ther. 5:785-791.

31. Rogers, B.E., Zinn, K.R., and Buchsbaum, D.J. 2000. Gene transfer strategies for improving radiolabeled peptide imaging and therapy. Q. J. Nucl. Med. 3:208-223.

32. Barrio, J.B., et al. 1989. 3-( $2^{\prime}-\left[{ }^{18} \mathrm{~F}\right]$ fluoroethyl $)$ spiperone: in vivo biochemical and kinetic characterization in rodents, nonhuman primates, and humans. Journal of Cerebral Metabolism and Blood Flow. 9:830-839.

33. Hemminki, A., et al. 2001. An adenovirus with enhanced infectivity mediates molecular chemotherapy of ovarian cancer cells and allows imaging of gene expression. Mol. Ther. 3:223-231.

34. Moon, D.H., et al. 2001. Correlation between 99mTc-pertechnetate uptakes and expressions of human sodium iodide symporter gene in breast tumor tissues. Nucl. Med. Biol. 7:829-834.

35. Jacobs, A., et al. 2001. Positron-emission tomography of vector-mediated gene expression in gene therapy for gliomas. Lancet. 9283:727-729.

36. Demetri, G.D., et al. 2002. Efficacy and safety of imatinib mesylate in advanced gastrointestinal stromal tumors. N. Engl. J. Med. 347:472-480.

37. Van den Abbeele, A.D., for the GIST Collaborative PET Study Goup (Dana-Farber Cancer Institute, Boston, Massachusetts, USA, OSU, Portlat, Oregon, Helsinki University Central Hopital, Turku University Central Hospital, Finland, Novartis Oncology). 2001. F18-FDG-PET provides early evidence of biological response to ST1571 patients with malignant gastrontestinal stromal tumors (GIST). Proc. Am. Soc. Clin. Oncol. 20:362a.

38. Frauwirth, K.A., et al. 2002. The CD28 signaling pathway regulates glucose metabolism. Immunity. 6:769-777.

39. Peng, T., Golub, T.R., and Sabatini, D.M. 2002. The immunosuppressant rapamycin mimics a starvation-like signal distinct from amino acid and glucose deprivation. Mol. Cell. Biol. 15:5575-5584.

40. Jolly, D. 1994. Viral vector systems for gene delivery. Cancer Gene Ther. 1:51-64.

41. Qiao, J., et al. 2002. Tumor-specific transcriptional targeting of suicide gene therapy. Gene Ther. 9:168-175.

42. Hackman, T., et al. 2002. Imaging expression of cytosine deaminase herpes virus thymidine kinase fusion gene $(\mathrm{CD} / \mathrm{TK})$ expression with $\left[{ }^{124} \mathrm{I}\right]$ FIAU and PET. Molecular Imaging. 1:36-42.

43. Tjuvajev, J.G., et al. 1999. Imaging adenoviral-mediated herpes virus thymidine kinase gene transfer and expression in vivo. Cancer Res. 59:5186-5193.
44. Bennett, J.J., et al. 2001. Positron emission tomography imaging for herpes virus infection: implications for oncolytic viral treatments of cancer. Nat. Med. 7:859-863.

45. Jacobs, A., et al. 2001. Positron emission tomography-based imaging of transgene expression mediated by replication-conditional, oncolytic herpes simplex virus type 1 mutant vectors in vivo. Cancer Res. 61:2983-2995.

46. Merritt, J.A., Roth, J.A., and Logothetis, C.J. 2001. Clinical evaluation of adenoviral-mediated p53 gene transfer: review of INGN 201 studies. Semin. Oncol. 16(Suppl.):S105-S114.

47. Ercikan-Abali, E.A., et al. 1993. Translational regulation of the synthesis of dihydrofolate reductase. Adv. Exp. Med. Biol. 338:537-540.

48. Mayer-Kuckuk, P., et al. 2002. Cells exposed to antifolates show increased cellular levels of proteins fused to dihydrofolate reductase: a method to modulate gene expression. Proc. Natl. Acad. Sci. U. S. A. 6:3400-3405

49. Ray, P., et al. 2002. Non-invasive quantitative imaging of protein-protein interactions in living subjects. Proc. Natl. Acad. Sci. U. S. A. 5:3105-3110.

50. Luker, G.D., et al. 2002. Noninvasive imaging of protein-protein interactions in living animals. Proc. Natl. Acad. Sci. U. S. A. 10:6961-6966.

51. Rosenberg, S.A., et al. 1990. Gene transfer into humans: immunotherapy of patients with advanced melanoma, using tumor-infiltrating lymphocytes modified by retroviral gene transduction. N. Engl. J. Med. 323:570-578.

52. Rooney, C.M., et al. 1995. Use of gene-modified virus-specific T lymphocytes to control Epstein-Barr-virus-related lymphoproliferation. Lancet. 345:9-13.

53. Verzeletti, S., et al. 1998. Herpes simplex virus thymidine kinase gene transfer for controlled graft-versus-host disease and graft-versusleukemia: clinical follow-up and improved new vectors. Hum. Gene Ther. 9:2243-2251.

54. Bonini, C., et al.1997. HSV-TK gene transfer into donor lymphocytes for control of allogeneic graft-versus-leukemia. Science. 276:1719-1724.

55. Heslop, H., et al. 2000. Administration of neomycin resistance genemarked EBV-specific cytotoxic T-lymphocytes as therapy for patients receiving a bone marrow transplant for relapsed EBV-positive Hodgkin disease. Hum. Gene Ther. 11:1465-1475.

56. Adonai, N., et al. 2002. Ex vivo cell labeling with 64Cu-pyruvaldehydebis(N4-methylthiosemicarbazone) for imaging cell trafficking in mice with positron-emission tomography. Proc. Natl. Acad. Sci. U. S. A. 99:3030-3035.

57. Koehne, G., et al. 2003. Serial in vivo imaging of the targeted migration of human HSV-TK-transduced antigen-specific lymphocytes. Nat. Biotechnol. 21:405-513.

58. Jacobs, A., et al. 2001. Positron-emission tomography of vector-mediated gene expression in gene therapy for gliomas. Lancet. 358:727-729. 